\section{A case of hepatomegaly}

\section{Joshi, A Belgaumkar, V Ratnayake, A Quaglia, D Austin}

A female publican in her 60s presented to clinic with abnormal liver function tests: bilirubin $17 \mu \mathrm{mol} / \mathrm{l}$ (normal range (NR) 0-17 $\mu \mathrm{mol} / \mathrm{l}$ ), alanine transaminase (ALT) 73 IU/l (NR 0$70 \mathrm{IU} / \mathrm{l})$, alkaline phosphatase (ALP) 463 IU/l (NR 42-128 IU/l), $\gamma$ glutamyl transferase (GGT) $784 \mathrm{IU} / \mathrm{l}$ (NR 2-35 IU/ l), albumin $35 \mathrm{~g} / \mathrm{l}$ (NR 35-50 g/l), prothrombin time (PT) $11.0 \mathrm{~s}$ (NR 10-12 s). She was asymptomatic with no history of weight loss or recent travel. She consumed an estimated 20 units of alcohol per week and was not taking any regular medications. Past medical history was unremarkable. The patient's body mass index was $19 \mathrm{~kg} / \mathrm{m}^{2}$. On examination, a $10 \mathrm{~cm}$ non-tender liver was palpable. No stigmata of chronic liver disease were present. The full blood count, and urea and electrolytes were normal. Viral hepatitis serology was negative. Autoimmune antibody serology, serum copper, caeruloplasmin and ferritin were normal.

An ultrasound of the abdomen confirmed an enlarged liver, with no focal lesions. Flow within the portal vein and hepatic vein was normal. No abnormalities were demonstrated within the biliary tree. The patient went on to have a computed tomographic (CT) scan of the abdomen (fig 1).

The patient then underwent a percutaneous liver biopsy (fig 2) because of continuing liver function deterioration (bilirubin $13 \mu \mathrm{mol} / \mathrm{l}$, albumin $34 \mathrm{~g} / \mathrm{l}$, ALP 968 IU/l, ALT 25 IU/l, GGT 1067 IU/l, PT $11.7 \mathrm{~s})$.

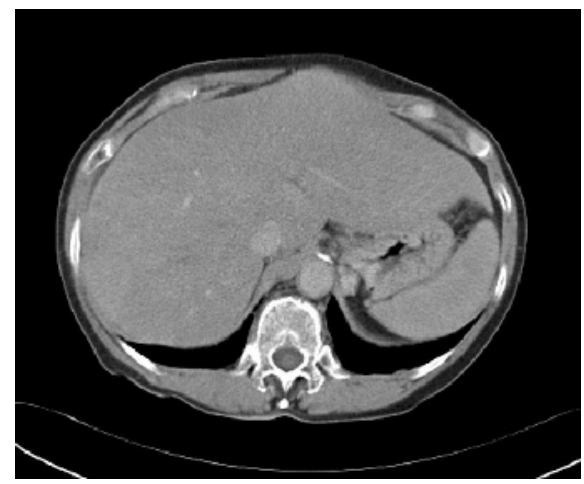

Figure 1 Computed tomographic scan of the abdomen.

\section{QUESTIONS}

(1) Comment upon the liver function tests and CT findings

(2) Given the liver biopsy findings, what is the diagnosis?

(3) What further investigations are required and what is the management of this disease?

\section{SELF-ASSESSMENT ANSWERS} A case of hepatomegaly

(1) Comment upon the liver function tests and CT findings

The liver function tests demonstrate a cholestatic picture with no evidence of a transaminitis or jaundice. Synthetic liver function appears preserved (normal serum albumin and PT). The CT scan of the abdomen (fig 1) demonstrates gross hepatomegaly without any focal lesions or biliary tree abnormalities. The spleen is of normal size, and there are no ascites present. Cholestatic liver function tests in the presence of hepatomegaly and the absence of alcohol excess would suggest an infiltrative process.

(2) Given the liver biopsy findings, what is the diagnosis?

The liver biopsy (fig 2) demonstrated a waxy amorphous material staining positive with Congo Red, therefore indicating amyloid deposition. A diagnosis of hepatic amyloidosis was made. The amyloid stained positive for $\lambda$ light chains, confirming a diagnosis of AL amyloidosis. Differentials of gross hepatomegaly include leukaemia and other reticulo-

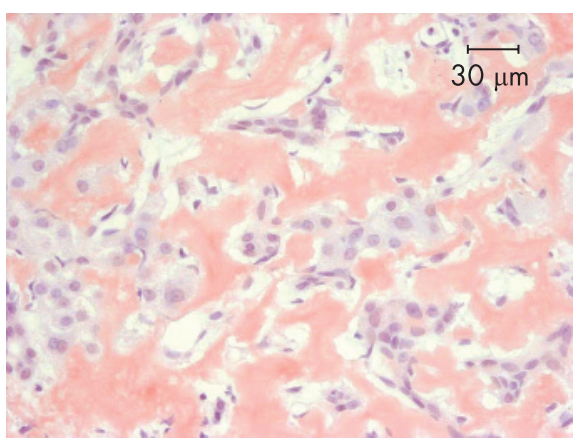

Figure 2 Percutaneous liver biopsy. endothelial disorders, sarcoidosis and malignancy (primary or secondary). Although the patient consumed more than the recommended units of alcohol per week (men 21 units per week, women 14 units per week), one would not expect this degree of hepatomegaly.

(3) What further investigations are required and what is the management of the disease?

A biopsy is required to confirm the diagnosis of amyloidosis. Organ specific biopsies (that is, liver, kidney, cardiac) have the greatest diagnostic yield, but have significant procedure related morbidity. When the diagnosis of amyloidosis is suspected, safer procedures include subcutaneous fat aspiration, rectal biopsies and bone marrow trephine. Assessment for an underlying plasma cell dyscrasia is required which includes serum electrophoresis and immuno-fixation of serum and urine together, looking for abnormal monoclonal light chains. Serum electrophoresis alone is inadequate as $20 \%$ of patients with amyloidosis will not have a detectable protein spike on the electrophoretic pattern. ${ }^{1}$ Immunofixation of serum and urine together looking for abnormal monoclonal light chains is $90 \%$ sensitive. ${ }^{1}$ Intravenous injection of technetium labelled serum amyloid P component (SAP) is both sensitive and specific for evidence of tissue amyloidosis deposits. ${ }^{2}$ An echocardiogram and urine analysis for proteinuria should be performed looking for cardiac and renal involvement, respectively. Management is aimed at preventing overproduction of precursor proteins, fibril formation, further tissue deposition and a negative balance of serum free light chains. This is achieved with various chemotherapy regimens which will be discussed below.

\section{DISCUSSION}

The patient was referred to the local haematology services for further assessment. Serum electrophoresis demonstrated a small $\operatorname{IgG} \lambda$ paraprotein band $(<3 \mathrm{~g} / \mathrm{l})$. Serum immunoglobulins were raised; IgG $17.2 \mathrm{~g} / \mathrm{l}$ (NR 6.0-16.0 g/l), IgA $7.0 \mathrm{~g} / \mathrm{l}$ (NR $0.8-4.0 \mathrm{~g} / \mathrm{l})$. Urine analysis was negative for Bence Jones protein. Raised serum free $\kappa$ and $\lambda$ light chains were found (34.2 mg/l (NR 3.3-19.4 mg/ l) and $90.56 \mathrm{mg} / \mathrm{l}(\mathrm{NR} 5.7 \mathrm{l}-26.30 \mathrm{mg} / \mathrm{l})$, respectively). A bone marrow aspirate did not show any evidence of myeloma or lymphoma. The bone marrow trephine confirmed the presence of amyloid, with an increase in plasma cells to $10 \%$. An IgG $\lambda$ secreting plasma cell dyscrasia was identified as the cause of the amyloidosis. The patient underwent four cycles of CTD 
(thalidomide, dexamethasone and cyclophosphamide) with good clinical response. The serum paraprotein dropped to $<\mathrm{lg} / \mathrm{l}$, and serum free light chain analysis showed a normal $\kappa: \lambda$ ratio. Liver function tests also improved (ALP 450 IU/l, GGT 65 IU/1).

Twenty-three months after her initial referral, the patient was admitted with worsening renal function, ascites and heart failure. At this stage haemodialysis was thought to be inappropriate and she was transferred to a local hospice for further palliative care.

Amyloidosis is a spectrum of diseases characterised by the deposition of fibrils in extracellular tissue. An insoluble matrix is formed, the result of abnormal folding of circulating proteins and further assembly. ${ }^{3}$ Crucially, the deposits are resistant to proteolysis. The two major types of amyloidosis are AL (primary), related to plasma cell dyscrasias, and AA (secondary), associated with chronic inflammatory disorders. Familial amyloidosis is inherited in an autosomal dominant fashion, and is commonly caused by mutant transthyretin (ATTR).

Amyloid deposition can occur in a variety of organs including the kidneys, heart, liver, gastrointestinal tract and peripheral nerves. The central nervous system is spared. Pathogenicity is due to the volume effect on the infiltrated organ. Until there is significant organ dysfunction, symptoms are usually non-specific. The characteristic histological finding is a waxy amorphous material displaying the classical apple green birefringence under polarised light microscopy with Congo red staining.

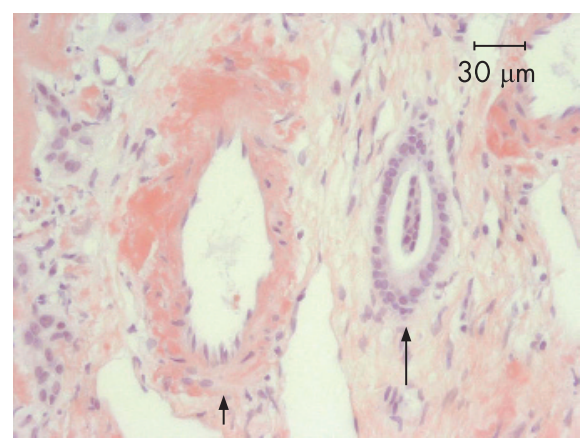

Figure 3 Amyloid deposition seen within the wall of a portal artery (short arrow). An interlobular bile duct is also present (long arrow).

\section{Learning points}

- Amyloidosis should be considered in patients with deranged liver function tests and hepatomegaly, in the absence of specific radiological findings

- Hepatic involvement in $\mathrm{AL} / \mathrm{AA}$ amyloidosis is common

- Stigmata of chronic liver disease and signs of portal hypertension are uncommon

- Alternative diagnostic tests to percutaneous liver biopsy are available

- Cardiac evaluation and assessment for proteinuria is important

The role and value of a liver biopsy in hepatic amyloidosis has been an issue of some debate. Although not contraindicated, there is an increased incidence of haemorrhage ( $2 \%$ ) post-biopsy ${ }^{4}$ and, rarely, hepatic rupture. Decreased synthesis of coagulation factors, factor $\mathrm{X}$ deficiency and amyloid infiltration of blood vessels are all thought to account for this increased incidence. ${ }^{3}$ Amyloid is usually deposited either within the peri-sinusoidal space of Disse, within vascular walls (fig 3), or in the perivascular portal tract fibrous tissue. ${ }^{5}$

The liver is commonly involved in $\mathrm{AL}$ and AA amyloidosis. ${ }^{5}$ Clinical features include hepatomegaly, a raised ALP and hyposplenism. The liver is classically described as "rock hard". 6 Varices and signs of portal hypertension are rare, as are stigmata of chronic liver disease. The level of hepatic involvement correlates poorly with serum liver function tests. Patients can present with fulminant liver failure, intrahepatic cholestatic jaundice and ascites, or intraparenchymal haemorrhage. $^{7}$ A poor prognosis is associated with an elevated bilirubin, congestive heart failure, and a platelet count $>500 \times 10^{9} /{ }^{4}{ }^{4}$

Treatment is aimed at preventing overproduction of precursor proteins, fibril formation and further tissue deposition.
Different chemotherapy regimens (cyclophosphamide, dexamethasone, and thalidomide, or vincristine, adriamycin and dexamethasone) have been used in $\mathrm{AL}$ amyloid. Management of organ specific failure is also important. Liver transplantation has been performed successfully in cases of rapidly progressive hepatic failure. Overall, median survival is between 8.5-9 months. ${ }^{4}$

\section{FINAL DIAGNOSIS}

AL hepatic amyloidosis.

Postgrad Med J 2007;83:e2 (http://www. postgradmedi.com/cgi/content/full/83/984/ e2).

doi: 10.1136/pgmi.2007.062471

\section{Authors' affiliations}

D Joshi, D Austin, Department of Gastroenterology, William Harvey Hospital, East Kent Hospitals, Ashford, Kent, UK A Belgaumkar, Digestive Disease Department, Brighton \& Sussex University Hospitals, Brighton, East Sussex, UK

V Ratnayake, Department of Haematology, William Harvey Hospital, East Kent Hospitals, Ashford, Kent, UK

A Quaglia, Department of Histopathology, Institute of Liver Studies, Kings College Hospital, London, UK

Correspondence to: Dr D Joshi, Department of Gastroenterology, William Harvey Hospital, East Kent Hospitals, Ashford, Kent, UK; djosh78@ hotmail.com

Received 18 June 2007

Accepted 18 July 2007

Competing interests: None

\section{REFERENCES}

1 Gertz MA, Merlini G, Treon SP. Amyloidosis and Waldenstrom's macroglobulinaemia. Haematology 2004; 1:257-82.

2 Hawkins, PN, Pepys, MB. Imaging amyloidosis with radiolabelled SAP. Eur J Nucl Med 1995;22:595.

3 Kacem C, Helali K, Puisieux F. Recurrent spontaneous hepatic rupture in primary hepatic amyloidosis. Ann Intern Med 1998; 129:339.

4 Park MA, Mueller PS, Kyle RA, et al. Primary (AL) hepatic amyloidosis: clinical features and natural history in 98 patients. Medicine 2003;82:291-8.

5 Brunt EM, Tiniakos DG. Metabolic storage diseases: amyloidosis. Clin Liver Dis 2004;8:915-30.

6 Gertz MA, Kyle RA. Hepatic amyloidosis: clinical appraisal in 77 patients. Hepatology 1997;25:118-21.

7 Gillmore JD, Lovat LB, Hawkins PN. Amyloidosis and the liver. J Hepatol 1999;30(suppl 1):17-23. 